Pacific Journal of Mathematics

ON A COHOMOLOGY THEORY BASED ON HYPERFINITE 


\title{
ON A COHOMOLOGY THEORY BASED ON HYPERFINITE SUMS OF MICROSIMPIEXES
}

\author{
RADE T. ŽIVALJEVIĆ
}

In this note we investigate a cohomology theory $H^{\#}(X, G)$, defined by M. C. McCord, which is dual to a homology theory based on hyperfinite chains of miscrosimplexes. We prove that if $X$ is a locally contractible, paracompact space then $H^{\#}(X, G) \simeq H_{\tilde{c}}^{\#}\left(X, \operatorname{Hom}\left(^{*} Z, G\right)\right)$ where $H_{\check{c}}^{\#}$ is the Čech theory. Nonstandard analysis, particularly the Saturation Principle, is used in this proof in essential way to construct a fine resolution of the constant sheaf $X \times \operatorname{Hom}(* Z, Z)$. This gives a partial answer to a question of McCord. Subsequently, we prove a proposition from which it is deduced that $\operatorname{Hom}\left({ }^{*} Z, Z\right)=\{0\}$ i.e. $H^{\#}(X, Z)=\{0\}$ if $X$ is paracompact and locally contractible. At the end we briefly discuss a related cohomology theory which is obtained by application of the internal (rather than external) $\operatorname{Hom}(\cdot, G)$ functor.

Introduction. As it is well known, nonstandard or infinitesimal analysis of Abraham Robinson was developed in an attempt to justify usage of infinitesimals and infinite numbers in calculus and other areas of mathematics. In the case of a general topological space $(X, \tau)$, a related notion is notion of the monad of $x \in X$, more precisely

$$
\operatorname{monad}(x)=\bigcap\{V \mid V \in \tau, x \in V\} .
$$

Informally, the monad of $x$ is the set of all $y \in{ }^{*} X$ which are infinitely close to $x$. This leads to a precise definition of a microsimplex. A $(n+1)$-tuple $s=\left(a_{0}, \ldots, a_{n}\right) \in\left({ }^{*} X\right)^{n+1}$ is a microsimplex if there exists $x \in X$ such that $\left\{a_{0}, \ldots, a_{n}\right\} \subset \operatorname{monad}(x)$.

Motivated by Vietoris homology and Alexander-Spanier cohomology, where the notion of a small simplex is used only in an informal sense, M. C. McCord in [7] defined a conceptually clear and technically easy homology theory based on hyperfinite chains of microsimplexes. The proofs are given in such a way that one automatically gets an associated cohomology theory by composing the chain complex functor with (external) functor $\operatorname{Hom}(\cdot, G)$.

At the end of his paper McCord raised three natural questions. The first two were about the relationship of his and Čech homology theory, whereas in the third a similar question is asked for his cohomology theory. The first two questions were answered by S. Garavaglia in [4]. He proved 
that McCord's theory is naturally isomorphic to Čech theory in the category of compact pairs. In the course of proof he obtained also the following remarkable result. Čech theory is exact if and only if the coefficient group is algebrically compact.

The main result of this paper is a partial answer to the third question of McCord. It turns out that McCord's cohomology with coefficients in $G$ is naturally isomorphic to Alexander-Spanier (or Čech) theory with coefficients in $\operatorname{Hom}\left({ }^{*} X, G\right)$ in the category of paracompact, locally contractible spaces! In the sequal we prove that $\operatorname{Hom}(* Z, Z)=\{0\}$ which means that McCord's cohomology with coefficients in $Z$ is trivial. At the end we briefly discuss a cohomology theory based on the application of internal (rather than external) $\operatorname{Hom}(\cdot, G)$ functor where $G$ is an internal group.

The reader is supposed to be acquainted with basic facts and methods of nonstandard analysis. Short introduction which covers all essential definitions and facts can be found in papers of Cutland [2] and Loeb [6] whereas more detailed and comprehensive introductions are Robinson [9], Davis [3] and Stroyan-Luxemburg [11]. Also, we assume some basic knowledge of the theory of sheaves. The main sources of information are Godement [5] and Bredon [1]. More precisely, all we need is a consequence of the Fundemantal Theorem in Godement [5] p. 178. Nonstandard analysis is used in an essential way (particularly the Saturation Principle) to construct a fine resolution of the constant sheaf $X \times$ $\operatorname{Hom}\left({ }^{*} Z, G\right)$. So, we assume that the nonstandard model is polysaturated.

\section{The relationship between McCord's and Čech cohomology.}

Definition 1.1. Let $(X, \tau)$ be a Hausdorff topological space. For $x \in X$, the monad of $x$ is the set $m(x)=\bigcap\left\{{ }^{*} O \mid x \in O\right.$ and $\left.O \in \tau\right\}$. Let us denote by $\mathrm{st}(\cdot)$ the standard part map, i.e. the function st:ns $\left({ }^{*} X\right) \rightarrow X$ where $\mathrm{ns}\left({ }^{*} X\right)=\left\{z \in{ }^{*} X \mid(\exists x \in X) z \in m(x)\right\}$ and $\operatorname{st}(z)$ is the unique $x \in X$, called the standard part of $z$, such that $z \in m(x)$. A point $z \in \mathrm{ns}\left({ }^{*} X\right)$ is called near-standard while the set $\mathrm{ns}\left({ }^{*} X\right)$ is called the set of near-standard points in $* X$. Two near-standard points are infinitely close if they belong to the same monad.

Definition 2.1. A simplex, or more precisely a $p$-simples, $p \in N$, in a set $S$ is just an ordered $(p+1)$-tuple of elements in $S$. If $X$ is a topological space then a microsimplex in ${ }^{*} X$ is a simplex in $\left.\operatorname{ns}^{*} X\right)$ with all vertices infinitely close to each other. Equivalently, a simplex $s=$ $\left(a_{0}, \ldots, a_{p}\right) \in\left({ }^{*} X\right)^{p+1}$ is a microsimplex if there exists $x \in X$ such that $a_{i} \in m(x)$ for all $i, 0 \leq i \leq p$. 
Let us recall the definition of McCord's homology groups with coefficients in $Z$. Let ${ }^{*}$ Free $\left(X^{p+1}\right)$ be the ${ }^{*}$-free Abelian group generated by the set $\left({ }^{*} X\right)^{p+1}$ of all $p$-simplexes in ${ }^{*} X$. The sequence of groups $\left\{{ }^{*}\right.$ Free $\left.\left(X^{p+1}\right), p \in N\right\}$ together with the boundary homomorphism $\partial_{p}$ : ${ }^{*}$ Free $\left(X^{p+1}\right) \rightarrow{ }^{*}$ Free $\left(X^{p}\right)$ which is defined in the usual way by

$$
\partial_{p}\left(\sum_{i=1}^{H} n_{i}\left(a_{0}^{i}, \ldots, a_{p}^{i}\right)\right)=\sum_{i=1}^{H} \sum_{j=0}^{p}(-1)^{j} n_{i}\left(a_{0}^{i}, \ldots, \hat{a}_{j}^{i}, \ldots, a_{p}^{i}\right)
$$

is an acylic chain complex. As usual, by $\left(a_{0}, \ldots, \hat{a}_{j}, \ldots, a_{p}\right)$ we denote the $(p-1)$-simplex obtained by deleting vertex $a_{j}$ of the $p$-simplex $\left(a_{0}, \ldots, a_{p}\right)$. Also, let us emphasize that all sums are formal and that $H$ and $n_{i}, 1 \leq i \leq H$, are hyperintegers. Microchain complex of $X$, or shortly $\mu$-complex of $X$, is

$$
\begin{aligned}
& M_{p}(X) \\
& =\left\{\sum_{i=1}^{H} n_{i} s_{i} \in{ }^{*} \operatorname{Free}\left(X^{p+1}\right) \mid s_{i} \text { is a microsimplex in } * X, 1 \leq i \leq H\right\}
\end{aligned}
$$

By applying the (external) functor $\operatorname{Hom}(\cdot, G)$ we obtain the corresponding cochain complex $M^{p}(X, G)=\operatorname{Hom}\left(M_{p}(X), G\right)$. More generally, the last definition can be extended to pairs of spaces $(X, A)$ where $A$ is a closed subspace, as follows. If $f: X \rightarrow Y$ is a continuous map then, by the nonstandard characterization of continuity, ${ }^{*} f$ sends monads of $* X$ into monads of ${ }^{*} Y$, hence it induces a chain map $M_{\#}(f): M_{\#}(X) \rightarrow M_{\#}(Y)$ where $M_{\#}(X)$ is an abbreviation for the $\mu$-complex of $X$. In particular, if $A \subset X$ is a closed subspace of $X$ and $i: A \rightarrow X$ the inclusion map, the exact sequence $0 \rightarrow M_{\#}(A) \rightarrow M_{\#}(X) \rightarrow M_{\#}(X, A) \rightarrow 0$ where $M_{\#}(X, A) \stackrel{\text { def }}{=} M_{\#}(X) / M_{\#}(A)$ splits which enables us to prove the exactness of the dual sequence

$$
0 \rightarrow M^{\#}(X, A ; G) \rightarrow M^{\#}(X, G) \rightarrow M^{\#}(A, G) \rightarrow 0
$$

where

$$
M^{\#}(X, A ; G) \stackrel{\text { def }}{=} \operatorname{Hom}\left(M_{\#}(X, A) ; G\right) .
$$

Our aim is to characterize the homology groups of the complex $M^{\#}(X, A ; G)$.

THEOREM 1.1. Let $X$ be a locally contractible, paracompact space and $G$ a group. If $H_{\check{c}}^{\#}(X, G)$ denotes the Čech cohomology functor whereas $H^{\#}(X, G)$ is the functor defined above then

$$
H^{\#}(X, G)=H_{\check{c}}^{\#}(X, \operatorname{Hom}(* Z, G)) \text {. }
$$


Proof. We need the following important result from the Theory of Sheaves (see Godement [5], p. 178, or Bredon [1]). Let

$$
0 \rightarrow \mathscr{F} \rightarrow \mathscr{L}_{0} \rightarrow \mathscr{L}_{1} \rightarrow \cdots \rightarrow \mathscr{L}_{n} \rightarrow \cdots
$$

be a fine or more generally a soft resolution of a sheaf $F$. Then $H^{n}(X ; \mathscr{F})$ $\cong H^{n}\left(\Gamma\left(X, \mathscr{L}_{\#}\right)\right)$ where the left term is the cohomology of $X$ with coefficients in $\mathscr{F}$ while the right term is the $n$th cohomology group of the cochain complex

$$
0 \rightarrow \Gamma\left(X, \mathscr{L}_{0}\right) \rightarrow \Gamma\left(X, \mathscr{L}_{1}\right) \rightarrow \cdots \rightarrow \Gamma\left(X, \mathscr{L}_{n}\right) \rightarrow \cdots
$$

where $\Gamma(X, \mathscr{G})$ is the group of all (global) sections of a sheaf $\mathscr{G}$. Our goal is to compute $H^{\#}(X, G) \stackrel{\text { def }}{=} H^{\#}\left(\operatorname{Hom}\left(M_{\#}(X), G\right)\right)$ so, in order to apply the theorem above we should realize the cochain complex $\operatorname{Hom}\left(M_{\#}(X), G\right)$ as a cochain complex of the form (1). Let $\mathscr{H}_{n}$ be the presheaf of groups on $X$ defined by $\mathscr{H}_{n}(U) \stackrel{\text { def }}{=} \operatorname{Hom}\left(M_{n}(U), G\right)$ and let $\mathscr{L}_{n}$ be the associated sheaf. Let us observe that $\Gamma\left(X, \mathscr{L}_{n}\right) \cong \mathscr{H}_{n}(X) \cong \operatorname{Hom}\left(M_{n}(X), G\right)$. Indeed, if $g \in \Gamma\left(X, \mathscr{L}_{n}\right)$ is given then $g$ is represented by a cover $\mathscr{U}$ of $X$ and a compatible family of maps $\left\{f_{U} \in \operatorname{Hom}\left(M_{n}(U), G\right) \mid U \in \mathscr{U}\right\}$. In order to define $f \in \operatorname{Hom}\left(M_{\#}(X), G\right)$ which extends all functions $f_{U}$, $U \in \mathscr{U}$ let us express an element $s \in M_{n}(X)$ as a finite sum $s=s_{1}+s_{2}$ $+\cdots+s_{m}$ such that for some $U_{i} \in \mathscr{U} s_{i} \in M_{n}\left(U_{i}\right)$. To see that the expression above is possible let us assume that $\mathscr{U}$ is a locally finite covering. Then, by the shrinking lemma for normal spaces, there exists a closed covering $\mathscr{K}$ of $X$ which is a refinement of $\mathscr{U}$ such that $\{\operatorname{int}(F) \mid F$ $\in \mathscr{K}\}$ is also a covering of $X$. The family of internal sets $\left\{{ }^{*} F \mid F \in \mathscr{K}\right\}$ covers all vertices of all microsimplexes in $s$ so (Saturation Principle) there exists a finite family $\left\{F_{1}, \ldots, F_{m}\right\}$ with the same property. Now, by repeating essentially the argument of the proof of Excision axiom in [7], it is easy to split $s$ in the sum $s=s_{1}+\cdots+s_{m}$ such that $s_{i} \in M_{n}\left(F_{i}\right)$. Indeed, if $s=\sum_{j=1}^{H} n_{i} \cdot d_{i}$ where $d_{j}=\left(a_{0}^{j}, \ldots, a_{n}^{j}\right) \in M_{n}(X)$, then

$$
s_{i}=\sum\left\{n_{j} d_{j} \mid d_{j} \in M_{n}\left(F_{i}\right) \& d_{j} \notin M_{n}\left(F_{k}\right) \text { for } k<i\right\} .
$$

Let us define the desired function $f \in \operatorname{Hom}\left(M_{n}(X), G\right)$ by the formula $f(s)=f_{U_{1}}\left(s_{1}\right)+\cdots+f_{U_{m}}\left(s_{m}\right)$ where $U_{i} \in \mathscr{U}$ and $F_{i} \subset U_{i}$ for all $i, 1 \leq i$ $\leq m$. To prove that $f$ is a well-defined homomorphism, it is enough to note that if $s=s_{1}+\cdots+s_{m}=s_{1}^{\prime}+\cdots+s_{n}^{\prime}$ then both expressions for $s$ can be refined by a third expression.

Let us prove now that $\mathscr{L}_{n}$ is a fine sheaf. Let $\mathscr{U}$ be a locally finite open covering of $X$ and $\mathscr{K}$ a closed shrinking of $U$ such that $\{\operatorname{int}(F) \mid F$ $\in \mathscr{K}\}$ is still a covering of $X$. Let $\phi$ be a function which to any $n$-simplex 
$s=\left(a_{0}, \ldots, a_{n}\right) \in X^{n+1}$ assigns, if it exists, a set $F \in \mathscr{K}$ such that $s \in F^{n+1}$. If $F \in \mathscr{K}$ is contained in $U \in \mathscr{U}$, let $e_{U}: \mathscr{L}_{n} \rightarrow \mathscr{L}_{n}$ be defined by

$$
e_{U}(f)(s)=f\left(s^{\prime}\right) \quad \text { where } f \in \operatorname{Hom}\left(M_{n}(V), G\right)
$$

represents a germ in $\mathscr{L}_{n}, s=\sum_{i=1}^{H} n_{i} s_{i} \in M_{n}(V)$ and $s^{\prime}$ is defined by $s^{\prime}=\sum\left\{\left.n_{j} \cdot s_{j}\right|^{*} \phi\left(s_{j}\right)=F\right\}$. Since $s^{\prime}$ is a chain in $F \subset U$ it is clear that the support of the endomorphism $e_{U}$ is in $U$. To see that $\left\{e_{U} \mid U \in \mathscr{U}\right\}$ is a partition of unity, i.e. that $\left\{U \mid e_{U}(f) \neq 0\right\}$ is finite and

$$
f=\sum\left\{e_{U}(f) \mid U \in \mathscr{U}\right\}
$$

let $V$ be small enough so that $\{U \mid U \cap V \neq \varnothing\}$ is finite. Hence, if $s=$ $\sum_{i=1}^{H} n_{i} \cdot s_{i} \in M_{n}(V)$ the set $\left\{{ }^{*} \phi\left(s_{j}\right) \mid j\right\} \subset\{U \in \mathscr{U} \mid U \cap V \neq \varnothing\}$ is finite and obviously $f(s)=\sum\left\{e_{U}(f)(s) \mid U \in \mathscr{U}\right\}$.

Let us prove now that

$$
0 \rightarrow \mathscr{F} \stackrel{j}{\rightarrow} \mathscr{L}_{0} \stackrel{d}{\rightarrow} \mathscr{L}_{1} \stackrel{d}{\rightarrow} \cdots \rightarrow \mathscr{L}_{n} \rightarrow \cdots
$$

is an exact sequence of sheaves where $\mathscr{F}=X \times \operatorname{Hom}(* Z, G)$ is a constant sheaf. Let $n \neq 0$ and let $V$ be a contractible neighborhood of $x \in X$. It is known (see [7]) that there exists a chain homotopy $D_{n}: M_{n}(V) \rightarrow M_{n+1}(V)$ such that $(n>0) s=\partial D_{n}(s)+D_{n-1} \partial(s)$ for any $s \in M_{n}(V)$. If $f \in$ $\operatorname{Hom}\left(M_{n}(V), G\right)$ is a cocycle then $f=d(g)$ where $g=f \circ D_{n-1}$, hence $f$ must be a coboundary. Now, let $n=0$. The map $j: \mathscr{F} \rightarrow \mathscr{L}_{0}$ is defined as follows. The map $X$ into a one point space induces a homomorphism $M_{0}(X) \rightarrow M_{0}(\{1\})={ }^{*} Z$ and $j: \operatorname{Hom}\left({ }^{*} Z, G\right) \rightarrow \operatorname{Hom}\left(M_{0}(X), G\right)$ is defined by applying the functor $\operatorname{Hom}(\cdot, G)$. Clearly, $d \circ j=0$. Now, let $V$ be a path connected open set in $X$ (recall that $X$ is locally contractible, hence locally path connected) and $f \in \operatorname{Hom}\left(M_{0}(V), G\right)$ such that $d(f)=$ 0 . Now, if $x, y \in M_{0}(V)$ are 0 -simplexes (i.e. they are points in $\mathrm{ns}\left({ }^{*} V\right)$ ) then there exists a 1-chain $s \in M_{1}(V)$ such that $y-x=\partial(s)$, hence $H \cdot y-H \cdot x=\partial(H \cdot s)$ for any $H \in{ }^{*} Z$. Hence, $f(H \cdot y)-f(H \cdot x)=$ $f(\partial(H \cdot s))=0$ which means that $f=j(g)$ where $g(H) \stackrel{\text { def }}{=} f(H \cdot x)$ for some $x \in \mathrm{ns}\left({ }^{*} V\right)$.

This completes the proof of the theorem because $H^{n}(X, \mathscr{F})$ is the Čech (or Alexander-Spanier) cohomology of $X$ with coefficients in $\operatorname{Hom}(* Z, G)$.

Corollary 1.1. Let $(X, A)$ be a pair of paracompact, locally contractible spaces such that $A$ is a closed subspace of $X$. Then

$$
H^{n}(X, A ; G)=H_{\check{c}}^{n}(X, A ; \operatorname{Hom}(* Z, G))
$$


Proof. The proof of this result follows directly from the naturality of the isomorphism between $H^{n}(X, G)$ and $H_{\check{c}}^{n}\left(X, \operatorname{Hom}\left({ }^{*} Z, G\right)\right)$ and the five lemma applied to the corresponding long exact sequences of the pair $(X, A)$ for both $H^{n}$ and $H_{\check{c}}^{n}$.

In light of the Theorem 1.1. it is interesting to determine the group $\operatorname{Hom}(* Z, G)$. Since ${ }^{*} N$ is a model of full arithmetic, in particular a model of Peano arithmetic, a partial answer is given by the following proposition which may be of some independent interest.

Proposition 1.1. Let $M$ be a nonstandard model of Peano arithmetic and $W$ the symmetrization of $M$ i.e. $W$ is the group obtained from the semigroup $M$ in the same way the group $Z$ is obtained from $N$. Then

(a) $\operatorname{Hom}(W, Z)=\{0\}$

(b) $\operatorname{Hom}(W, Z / n Z)=Z / n Z, n \neq 0$

(c) $\operatorname{Hom}(W, W) \supsetneqq W$.

Proof. (a) Let $f: W \rightarrow Z$ be a homomorphism and $i: Z \rightarrow W$ the inclusion map. Let us note that $W / Z$ is divisible by any $n \in Z /\{0\}$. As a consequence $f(Z)=f(W)$, otherwise $f$ would induce a homomorphism from a divisible group $W / Z$ onto a nontrivial cyclic group $f(W) / f(Z)$. If $f(Z)$ is nontrivial it can be assumed without loss of generality that $f(Z)=Z$ and $f \mid Z=1_{Z}$ i.e. we can assume that $i: Z \rightarrow W$ splits the exact sequence $0 \rightarrow \operatorname{Ker}(f) \rightarrow W \stackrel{f}{\rightleftarrows} Z \rightarrow 0$. In other words $W=Z \oplus D$ where $D=\operatorname{Ker}(f)$ is a divisible ${ }^{i}$ group. Let $P$ be an infinite prime number in $W$ of the form $5 k+3$. The existance of $P$ follows from the fact that there exist infinitely many primes of the form $5 k+3$ (Dirichlet thm., see [10]) and the fact that every nonstandard model of Peano arithmetic is $\Sigma_{m}$-recursively saturated. In particular the following type

$$
\{x \text { is a prime number }\} \cup\{(\exists k) x=5 k+3\} \cup\{x \geq \underline{n} \mid n \in N\}
$$

where $\underline{n}(n \in N)$ are numerals, is realized in any nonstandard model of Peano arithmetic. It is interesting to note that the Dirichlet Theorem itself is a theorem of Peano arithmetic. Indeed, G. Takeuti in [12] showed that the results of classical number theory which are obtained with the aid of so called elementary complex analysis are actually provable in Peano arithmetic.

Now, let $f(P)=n \in Z$ i.e. $P=n+(P-n)$ where $P-n \in D$. Since $P$ is a prime number and $P-n$ is divisible by any nonzero integer we see that $n=1$ or $n=-1$. This is a contradiction because in both cases $P-n$ cannot be divisible by 5 . 
(b) It is enough to show that $\operatorname{Hom}(W, Z / n Z)=\operatorname{Hom}(Z, Z / n Z)$, i.e. that for any two homomorphisms $f, g \in \operatorname{Hom}(W, Z / n Z) f|Z=g| Z$ implies $f=g$. This is true because $h=f-g$ induces a homomorphism from a divisible group $W / Z$ into a cyclic group so it must be trivial.

(c) $W$ is imbedded in $\operatorname{Hom}(W, W)$ in the obvious way. To show that this inclusion $W \subset \operatorname{Hom}(W, W)$ is proper let

$$
A=\{x \in W \mid x \text { is divisible by any } n \in Z \backslash\{0\}\} .
$$

$A$ is a nontrivial divisible group so both $A$ and $W / Z$ can be thought of as vector spaces over $Q$. Any nontrivial $Q$-linear map from $W / Z$ to $A$ induces a homomorphism in $\operatorname{Hom}(W, W)$ which is zero on $Z$.

2. Yet another cohomology theory. Instead of applying the standard external $\operatorname{Hom}(\cdot, G)$ functor to the complex $M_{\#}(X)$, one may find it more natural to apply a functor which is based on the internal $\operatorname{Hom}_{\text {int }}(\cdot, G)$ functor where $G$ is internal group and $\operatorname{Hom}_{\text {int }}(A, G) \stackrel{\text { def }}{=}\{f: A \rightarrow G \mid f$ is an internal homomorphism $\}$. We shall briefly outline this construction. A different, although similar in spirit, construction was proposed by J. P. Reveilles [8] but in the context of E. Nelson's Set Theory.

Definition 2.1. Let $\Delta_{p}$ be the set of all microsimplexes in ${ }^{*} X$. Let $M_{\mu}^{p}(X, G) \stackrel{\text { def }}{=}\left\{g: \Delta_{p} \rightarrow G \mid \exists f:\left({ }^{*} X\right)^{p+1} \rightarrow G\right.$, internal, such that $g=$ $\left.f \mid \Delta_{p}\right\}$. Then $\left\{M_{\mu}^{\#}(X, G), d\right\}$ where $d(g)(s)=f(\partial s)$ is a cochain complex. Let us note that every internal function $f:\left({ }^{*} X\right)^{p+1} \rightarrow G$ can be identified with a homomorphism from ${ }^{*}$ Free $\left(X^{p+1}\right)$ to $G$ i.e. $d(g)$ is well defined. More generally, if $i: A \rightarrow X$ is the inclusion map then $M_{\mu}^{\#}(X, A ; G) \stackrel{\text { def }}{=} \operatorname{Ker}\left\{M^{\#}(X, G) \rightarrow M^{\#}(A, G)\right\}$. The cohomology groups of the chain complex $M_{\mu}^{\#}(X, A ; G)$ are denoted by $H_{\mu}^{\#}(X, A ; G)$. It is not difficult to check that all axioms of Eilenberg and Steenrod for a cohomology theory are satisfied in this case.

THEOREM 2.1. If $X$ is a paracompact space and $G$ an internal group then $H_{\mu}^{n}(X, G)=H^{n}(X, G)$ where $H^{n}(X, G)$ is the Alexander-Spanier (or Čech) cohomology of the space $X$ with coefficients in $G$.

Proof. Since it is based on similar ideas as the proof of Theorem 1.1 we shall give only a brief outline of the proof. It is enough to define a fine resolution.

$$
0 \rightarrow \underline{G} \rightarrow \mathscr{F}_{0} \rightarrow \cdots \rightarrow \mathscr{F}_{n} \rightarrow \cdots
$$

of the constant sheaf $\underline{G}=X \times G$ such that $M_{\mu}^{n}(X, G)=\Gamma\left(X, F_{n}\right)$ for all 
$n \geq 0$. Let $F_{p}$ be a presheaf defined by $F_{p}(U)=\left\{f:\left({ }^{*} U\right)^{p+1} \rightarrow G \mid f\right.$ internal $\}$ and let $\mathscr{F}_{p}$ be the associated sheaf. To prove the desired isomorphism one has to show that not only every $f \in M^{p}(X, G)$ induces a section in $\mathscr{F}_{p}$ but that every section arises in this way. This is proved by an easy saturation argument by observing that for any compatible family $\left\{f_{U} \mid U \in \mathscr{U}\right\}$ of internal functions $f_{U}:\left({ }^{*} U\right)^{p+1} \rightarrow G$, where $\mathscr{U}$ is a locally finite open cover of $X$, there exists an internal function $f$ : $\left({ }^{*} X\right)^{p+1} \rightarrow G$ which extends all functions $f_{U}$. The proof that (2) is indeed a fine resolution of the constant sheaf $\underline{G}$ is similar to the proof of the corresponding result for Alexander-Spanier cohomology so we omit the details.

Acknowledgments. This paper is based on some results from the first Chapter of my $\mathrm{PhD}$. thesis "Infinitesimal Analysis and Homology Theory”, University of Wisconsin, Madison, 1985. I wish to express my thanks to my advisor Professor H. Jerome Keisler for his generous support and encouragement.

\section{REFERENCES}

[1] G. E. Bredon, Sheaf Theory, McGraw-Hill, New York 1967.

[2] N. J. Cutland, Nonstandard measure theory and its applications, Bull. London Math. Soc., 15 (1983), 529-589.

[3] M. Davis, Applied Nonstandard Analysis, J. Wiley Pub., New York, 1977.

[4] S. Garavaglia, Homology with equationally compact coefficients, Fund. Math., 100 (1978), 89-95.

[5] R. Godement, Topologie Algébrique et Théorie des Faisceaux, Hermann, Paris (1958).

[6] P. A. Loeb, An Introduction to Nonstandard Analysis and Hyper-finite Probability Theory, Probalistic Analysis and Related Topics, Vol. 2 (Ed. A. T. Bharucha-Reid, Academic Press, New York, 1979) pp. 105-142.

[7] M. C. McCord, Non-standard analysis and homology, Fund. Math., 74 (1972), 21-28.

[8] J. P. Reveilles, Infinitesimaux et Topologie, Publ. I.R.M.A., Strasbourg, 1983.

[9] A. Robinson, Non-Standard Analysis, North-Holland, Amsterdam, 1966.

[10] J. P. Serre, A Course in Arithmetic, Springer-Verlag, New York, 1973.

[11] K. D. Stroyan, and W. A. J. Luxemburg, Introduction to the Theory of Infinitesimals, Academic Press, 1976.

[12] G. Takeuti, Two Applications of Logic to Mathematics, Princeton Univ. Press, 1978.

Received December 1, 1985.

MATHEMATICS INSTITUTE

KNEZ MiHailova $35 / 1$

11000 BEOGRAD

Yugoslavia

Temporary address: Western Illinois University

Macomb, IL 61455

U.S.A. 


\section{PACIFIC JOURNAL OF MATHEMATICS EDITORS}

\author{
V. S. VARADARAJAN \\ (Managing Editor) \\ University of California \\ Los Angeles, CA 90024 \\ HERBERT Clemens \\ University of Utah \\ Salt Lake City, UT 84112 \\ R. FINN \\ Stanford University \\ Stanford, CA 94305
}

HERMANN FLASCHKA

University of Arizona

Tucson, AZ 85721

RAMESH A. GANGOLLI

University of Washington

Seattle, WA 98195

VAughan F. R. JONES

University of California

Berkeley, CA 94720

ROBION KIRBY

University of California

Berkeley, CA 94720
C. C. MoOrE

University of California

Berkeley, CA 94720

H. SAMELSON

Stanford University

Stanford, CA 94305

HAROLD STARK

University of California, San Diego

La Jolla, CA 92093

\section{ASSOCIATE EDITORS}
R. AREnS
E. F. BECKENBACH
B. H. NEUMANN
F. WOLF
K. YOSHIDA (1906-1982)

\section{SUPPORTING INSTITUTIONS}

UNIVERSITY OF ARIZONA

UNIVERSITY OF BRITISH COLUMBIA

CALIFORNIA INSTITUTE OF TECHNOLOGY

UNIVERSITY OF CALIFORNIA

MONTANA STATE UNIVERSITY

UNIVERSITY OF NEVADA, RENO

NEW MEXICO STATE UNIVERSITY

OREGON STATE UNIVERSITY
UNIVERSITY OF OREGON UNIVERSITY OF SOUTHERN CALIFORNIA STANFORD UNIVERSITY UNIVERSITY OF HAWAII UNIVERSITY OF TOKYO UNIVERSITY OF UTAH WASHINGTON STATE UNIVERSITY UNIVERSITY OF WASHINGTON 


\section{Pacific Journal of Mathematics}

\section{Vol. 128, No. $1 \quad$ March, 1987}

Anthony Peter Bahri and Peter Gilkey, The eta invariant, $\operatorname{Pin}^{c}$ bordism, and equivariant $\operatorname{Spin}^{c}$ bordism for cyclic 2-groups $\ldots \ldots \ldots \ldots \ldots \ldots 1$

Friedrich-Wilhelm Bauer, Extensions of generalized homology theories . . . 25

Marilyn Breen, A characterization theorem for compact unions of two

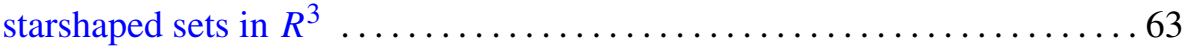

Boniface Ihemotuonye Eke, Special generating sets of purely inseparable extension fields of unbounded exponent $\ldots \ldots \ldots \ldots \ldots \ldots \ldots \ldots \ldots \ldots$

Robert William Gilmer, Jr. and William James Heinzer, Jónsson $\omega_{0}$-generated algebraic field extensions $\ldots \ldots \ldots \ldots \ldots \ldots \ldots \ldots \ldots . . .61$

Guido Lupacciolu, Holomorphic continuation in several complex variables

Douglas C. McMahon, Jaap C. S. P. van der Woude and Ta-Sun Wu,

Connectedness related to almost periodicity of compositions of flow

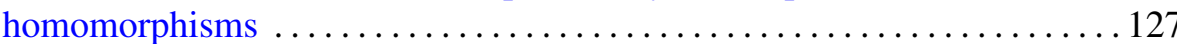

Sophocles Mercourakis, Some characterizations of analytic metric spaces

Peter Frederick Stiller, The Picard numbers of elliptic surfaces with many

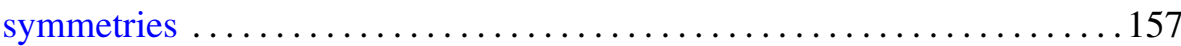

David J. Winter, Reducible complements of Lie algebra radicals ........ 191 Rade Živaljević, On a cohomology theory based on hyperfinite sums of microsimplexes 